\title{
Inversión con modelos Markov-Switching GARCH: un estudio comparativo entre México y Argentina
}

\author{
Investing with Markov-Switching GARCH models: \\ A comparative test between Mexico and Argentina
}

\author{
Oscar V. De la Torre-Torres* \\ Universidad Michoacana de San Nicolás de Hidalgo \\ Recibido el 29 de julio de 2019; aceptado el 18 de febrero de 2020 \\ Disponible en Internet el: 26 de febrero de 2020
}

\section{Resumen}

En este artículo se estudia el empleo de modelos markovianos con cambio de régimen (Markov-Switching) de dos regímenes, varianza GARCH y con funciones de verosimiltud gaussiana o t-Student homogéneas entre regímenes. Esto para administrar activamente portafolios en la bolsa de Buenos Aires y la Bolsa Mexicana de Valores. Al realizar 996 simulaciones semanales de enero del 2000 a enero del 2019, se ejecutó la siguiente estrategia de inversión para un portafolio denominado en dólares de los EEUU: 1) invertir en el activo libre de riesgo si la probabilidad de estar en el régimen de alta volatilidad en $\mathrm{t}+1$ es mayor a $50 \%$ o 2) invertir en el índice accionario en caso contrario. Los resultados sugieren que emplear modelos MS-GARCH t-Student en una administración activa lleva a un mejor desempeño en el caso argentino y los modelos MS con varianza constante y función gaussiana en el mexicano. Esto en comparación con una estrategia pasiva tipo "comprar y mantener".

\section{Código JEL: C580, G11, G170}

Palabras clave: Markov-Switching GARCH; Cadenas markovianas; Administración activa de portafolios; Bolsa de comercio de Buenos Aires; Bolsa Mexicana de Valores; Mercados frontera; Finanzas computacionales; Administración de riesgos

\footnotetext{
*Autor para correspondencia

Correo electrónico: odelatorre@umich.mx (O. V. De la Torre Torres).

La revisión por pares es responsabilidad de la Universidad Nacional Autónoma de México.

http://dx.doi.org/10.22201/fca.24488410e.2020.2657

0186- 1042/@ 2019 Universidad Nacional Autónoma de México, Facultad de Contaduría y Administración. Este es un artículo

Open Access bajo la licencia CC BY-NC-SA (https://creativecommons.org/licenses/by-nc-sa/4.0/)
} 


\begin{abstract}
In the present paper I test the benefits, for active portfolio management purposes, of using two-regime Markov-Switching (MS) models with GARCH variance. This, with either a Gaussian or t-Student homogeneous likelihood function, in the Buenos Aires and in the Mexican Stock Exchanges. By performing 996 weekly simulations from January 2000 to January 2019 in each MS model, I tested the next investment strategy for a U.S. dollar based investor: 1) to invest in the risk-free asset if the probability of being in the high-volatility regime at $\mathrm{t}+1$ is higher than $50 \%$ or 2 ) to do it in an equity index otherwise. The results suggest that the t-Student MS-GARCH model is the best option to generate alpha in Argentina and the constant variance gaussian one in Mexico. This, against a "buy and hold" investment strategy.
\end{abstract}

JEL code: C580, G11, G170

Keywords: Markov-Switching GARCH; Markov chain processes; Active portfolio management; Buenos Aires stock exchange; Mexican stock exchange; Frontier markets; Computational Finance, Risk management

\title{
Introducción
}

Una de las tareas de mayor sensibilidad en la práctica financiera, en específico la administración de inversiones y en la de riesgos, es cuantificar adecuadamente los parámetros estadísticos que se utilizarán como insumos para el proceso de toma de decisiones. Otra actividad sensible y relacionada a la anterior es el adecuado cálculo o inferencia del momento apropiado para invertir o desinvertir en un portafolio o cartera. Como se verá en el presente trabajo, el empleo de modelos markovianos con cambio de régimen (modelos Markov-Switching o MS de ahora en adelante) será de amplia utilidad para determinar los momentos apropiados de inversión y desinversión. Lo anterior partiendo del cálculo de una probabilidad $\xi_{-}(\mathrm{s}=2, \mathrm{t}) \mathrm{de}$ estar en un periodo, régimen o estado de la naturaleza conocido como de "alta volatilidad". Al determinar dicha probabilidad se logran, como parámetros de salida, tanto un rendimiento esperado como un nivel de exposición al riesgo que serán parte del cálculo de la verosimilitud previamente citada y que son también parámetros o insumos centrales para la toma de decisiones de inversión en la práctica financiera.

En la práctica profesional y en la academia se han empleado, para cuantificar el nivel de rendimiento esperado por la o el inversionista, métodos como son la media aritmética o la exponencial. De manera complementaria y como se verá en la revisión de literatura que motiva el presente trabajo, múltiples métodos de media condicional se han sugerido para fines de eficiencia computacional o para dar una mayor robustez a la cuantificación de estos parámetros (Alexander, 2002; Ang y Bekaert, 2002a; Sharpe, 1963, 1964), mismos que rea- 
lizan la cuantificación de un rendimiento medio condicionado, dado el valor que un factor o grupo de factores puede tener en $t$

Otras propuestas o extensiones a la cuantificación del rendimiento esperado se dan con el empleo de los modelos ARMA ${ }^{1}$ o ARMAX. En el primer caso, se establece que el proceso estocástico generador de la serie de tiempo de los rendimientos de un activo o valor $r_{t}$ depende, dadas situaciones diversas propias del activo modelado, tanto de valores pasados (rezagos) de los rendimientos como de rezagos en los residuales $\varepsilon_{t}$

$$
r_{t}=\alpha+\sum_{p=1}^{P} \beta_{p} \cdot r_{t-p}+\sum_{q=1}^{Q} \gamma_{p} \cdot \varepsilon_{t-1}+\varepsilon_{t}
$$

El modelo en (1) se conoce como un proceso ARMA (previamente definido en la nota al pie 1), observando que el segundo término de la expresión se conoce como el término AR o auto regresivo, el cual mide el impacto de valores pasados $\left(r_{i, t-p}\right)$ en el nivel de rendimiento actual $\left(r_{i, t}\right)$. El tercer término en (1), se conoce como MA o de medias móviles, mismo que mide el impacto que valores pasados de los residuales $\left(\varepsilon_{i, t-q}\right)$ de (1) tienen también en el valor de $r_{i, t}$.

Como punto de partida y para fines de cuantificación de rendimientos en el presente trabajo, se seguirá a la práctica de valuación de activos y a la literatura relacionada a la Econometría Financiera, consistente en cuantificar $r_{t}$ con el método de los rendimientos continuamente compuestos, mismo que parte del precio actual $\left(P_{i, t}\right)$ y pasado $\left(p_{i, t-1}\right)$ del activo, índice, moneda o mercancía analizado:

$$
r_{i, t}=\ln \left(P_{i, t}\right)-\ln \left(p_{i, t-1}\right)
$$

Lo anterior permite que la serie de tiempo en $r_{t}$ sea estacionaria ${ }^{2}$, dado el cálculo de la primera diferencia del logaritmo del precio. Partiendo de esta situación, (1) es la forma funcional de un modelo ARMA, cuyos pronósticos en $t+1$ son integrados ${ }^{3}$ al precio actual $\left(P_{t}\right)$. Esto a fin de pronosticar su precio en $p_{t+1}$.

Partiendo de la cuantificación del rendimiento esperado con alguno de los métodos previamente descritos, se observa que es de similar importancia el nivel de exposición al riesgo que comenzó a aproximarse, en las primeras etapas históricas de la práctica financiera

\footnotetext{
${ }^{1}$ Para fines de exposición, se utilizarán las siglas, en idioma inglés: ARMA (Auto Rregresive Moving Average o Auto Regresivo con Medias Móviles) and ARIMA (Auto Rregresive Integrated Moving Average o Auto Regresivo con Medias Móviles Integrado).

${ }^{2}$ La estacionariedad es una propiedad que deben tener las series de tiempo $r_{t}$ para poder realizar análisis econométrico con las mismas. Esta consiste en que los parámetros de localización (media) y escala (varianza) deben ser constantes en el tiempo o, de no ser posible, estables en su valor al largo del tiempo.

${ }^{3}$ De ahí la esencia y nombre del modelo ARIMA (p,I,q) que aplica para $p_{t}$, dado el modelo ARMA en (1).
} 
contemporánea, con la varianza convencional $\left(\sigma_{i, t}^{2}=\sum_{t=1}^{T}\left(r_{i, t}-\mu_{i}\right) \cdot N^{-1}\right)$. La limitante de este método de cálculo es que tiene un valor constante a lo largo del tiempo. Sin embargo, con las propuestas de Engle (1982) y Bollerslev (1987), se lograron avances significativos para cuantificar este parámetro, ya que la varianza puede ser estimada como un parámetro cambiante, la cual es una característica fundamental de los modelos $\mathrm{GARCH}^{4} \mathrm{y}$ algo propio de las series de tiempo financieras que no presentan volatilidades o varianzas constantes a lo largo del tiempo:

$$
\sigma_{i, t}^{2}=\sigma_{0}+\sum_{p=1}^{P} \beta_{p} \cdot \varepsilon_{i, t-p}^{2}+\sum_{q=1}^{Q} \gamma_{p} \cdot \sigma_{i, t-q}^{2}+v_{t}
$$

En la expresión previa y tal como se mencionó, el valor de la varianza $\sigma_{t}^{2}$ en $t$ se cuantifica por medio de los valores cuadráticos de los residuales, mismos que se determinan con la media o rendimiento esperado $\left(\varepsilon_{i, t-p}^{2}\right)$, así como de los valores pasados de la varianza $\left(\sigma_{i, t-q}^{2}\right)$ estimada en (3). El segundo y tercer términos en (3) se conocen como ARCH y GARCH respectivamente. Dado esto, se dice que el modelo es uno ARCH cuando (3) tiene esta forma funcional: $\sigma_{t}^{2}=\sigma_{0}+\sum_{p=1}^{P} \beta_{p} \cdot \varepsilon_{i, t}^{2}+v_{t}$ o se conoce como GARCH cuando se expresa (3) de manera completa.

El método de cuantificar riesgo o varianzas de manera dinámica a lo largo del tiempo por medio de modelos GARCH permitió grandes avances en la industria financiera. Más específicamente, los avances se lograron en áreas como es la valuación de activos y la administración de riesgos. Esto es así debido a que, en tiempos de crisis o alta volatilidad (fluctuación de precios) financiera, se miden varianzas altas y, en los periodos "normales" o de baja volatilidad, niveles bajos en la misma.

Partiendo de esta perspectiva de los modelos GARCH, múltiples extensiones se han propuesto a los mismos. Entre las más empleadas y reconocidas se encuentran el modelo exponencial de efectos asimétricos EGARCH de Nelson (1991) o el GJR-GARCH con apalancamiento de residuales negativos de Glosten, Jaganathan and Runkle (1993). Hay incluso otras extensiones con funciones de verosimilitud diversas (las cuales no se mencionarán aquí, dada la amplitud de la revisión).

Una propiedad útil del modelo GARCH como el presentado en (3) es el hecho de que la suma $\sum_{p=1}^{P} \beta_{p}+\sum_{q=1}^{Q} \gamma_{p}$ lleva a un concepto conocido como "persistencia", lo que implica que, si $\sum_{p=1}^{P} \beta_{p}+\sum_{q=1}^{Q} \gamma_{p} \approx$ en $(3)$, los niveles de volatilidad alta "persistirán" por periodos

\footnotetext{
${ }^{4}$ Siglas de los modelos Generalizados con Heteroscedasticidad Auto Regresiva Condicionada (Generalized AutoRregresive Contitional Heteroskedasticity).
} 
largos en el tiempo. Esta situación es algo que puede presentarse en series de tiempo financieras como las generadas con (2). Como una de las múltiples explicaciones dadas a este resultado, Dueker (1997), Lamoureoux and Lastrapes (1990); Hamilton and Susmel (1994), Klaassen (2002) y Hass, Mitnik and Paoella (2004) proponen que la alta persistencia se debe a que el proceso estocástico de la serie de tiempo no debe modelarse de manera unimodal. Esto es, con una media y desviación estándar (y, por ende, una sola función de probabilidad), sino con múltiples medias y desviaciones estándar, que son propias de una función de probabilidad múltimodal. Esto lleva a concebir a la serie de tiempo como una que tienes $s-1(\operatorname{con} s=1,2, \ldots, S)$ cambios estructurales que llevan a la presencia no de uno, sino de regímenes o estados de la naturaleza en el comportamiento de $r_{t}$.

Por esta razón y gracias a las propuestas de Hamilton $(1989,1994)$, el rendimiento y exposición al riesgo (varianza) esperados pueden aproximarse por medio de un modelo de cambios markovianos (MS) de estados. Dado lo anterior, el modelo ARMA en (1) puede extenderse como sigue:

$$
r_{i, t}=\alpha_{s}+\sum_{p=1}^{P} \beta_{p, s} \cdot r_{i, t-p}+\sum_{q=1}^{Q} \gamma_{p, s} \cdot \varepsilon_{t-1}+\varepsilon_{t}
$$

Dado el vector de parámetros $\theta=\left[\alpha_{s}, \beta_{s}, \gamma_{s}\right]$, el modelo MS permite, como parámetro de salida, inferir la probabilidad $\xi_{s, t}$ de estar en el régimen $S$ en $t$. Esto junto con las probabilidades de transición $\left(\pi_{i, j}=P\left(s_{t}=i \mid s_{t-1}=j, \theta, r_{t}\right), \theta=\left[\alpha_{s}, \beta_{s}, \gamma_{s}, \sigma_{s}^{2}, \xi_{s, t}\right]\right)$ que estiman la verosimilitud de transitar de un régimen $s=i$ en $t$, a otro $s=j$ en $t+1$. Como se puede apreciar, este comportamiento es propio de una cadena markoviana latente y no observable de manera directa por la o el analista. Dado lo anterior, la representación matricial de estas probabilidades de transición se resume en lo que se conoce como la matriz de probabilidades de transición ח:

$$
\boldsymbol{\Pi}=\left[\begin{array}{ccc}
\pi_{i, i} & \ldots & \pi_{j, i} \\
\vdots & \vdots & \vdots \\
\pi_{i, j} & \cdots & \pi_{j, j}
\end{array}\right]
$$

Una limitante del modelo MS como el dado en (4) es que, desde su propuesta original, el mismo asume que la varianza (léase desviación estándar) de cada régimen es constante en el tiempo. Dado esto, una extensión al modelo MS es la realizada por Hamilton y Susmel (1994), en la que el modelo GARCH en (3), es ahora uno propio de $S$ regímenes. Es decir, un modelo GARCH con parámetros para cada régimen (MS-GARCH): 


$$
\sigma_{t}^{2}=\sigma_{0, s}+\sum_{p=1}^{P} \beta_{p, s} \cdot \varepsilon_{t-p}^{2}+\sum_{q=1}^{Q} \gamma_{p, s} \cdot \sigma_{t-q}^{2}+v_{t}
$$

Dada la naturaleza dinámica de los modelos MS y la posibilidad de inferir las probabilidades suavizadas y de transición para $S$ regímenes, muchas aplicaciones de estos modelos se han estudiado. El uso de los modelos MS en la toma de decisiones de inversión es la aplicación de interés en el presente trabajo y se desarrolla con la propuesta primigenia de Brooks y Persand, seguida de Ang y Bekaert (2002a, 2004). Estos autores sugieren el empleo de modelos MS en la toma de decisiones de índices accionarios del Reino Unido, así como el empleo de modelos MS para administrar portafolios internacionalmente diversificados. Siguiendo estos dos trabajos, también se puede encontrar el trabajo de Kritzman, Page y Turkington (2012), Hauptmann et. al. (2014) y de De la Torre, Galeana y Álvarez-García (2018) , siendo éste último una aplicación en mercados accionarios tanto desarrollados como en uno emergente ${ }^{5}$ (México).

Como se verá en la revisión de literatura que motiva el presente, hay algunas áreas de oportunidad que se busca solventar en el presente trabajo:

1. Los estudios previos sobre los beneficios de los modelos MS en el proceso de toma de decisiones de inversión no estudian el uso de modelos MS-ARCH o MS-GARCH en el proceso de toma de decisiones de inversión.

2. De los trabajos previos, enfocados a la toma de decisiones de inversión con modelos MS, solo uno se enfoca a países emergentes y latinoamericanos (México), dejando la oportunidad de estudiar su extensión a otros mercados latinoamericanos como es el caso de Argentina. Esto por medio del índice MSCI Argentina.

3. Poco se ha estudiado, en diferentes revisiones previas, sobre el beneficio o empleo de modelos MS, MS-ARCH o MS-GARCH en los países de interés no solo en áreas de inversión, sino en modelado en general. Los trabajos de Camacho y Pérez-Quirós (2014), Cabrera et. al. (2017) y Sosa, Ortiz y Cabello (2018) hacen interesantes modelados con dos y tres regímenes. De manera específica para los fines del presente trabajo, el trabajo Cabrera et. al. (2017) es el único que emplea modelos MS-GARCH en Latinoamérica. Dado esto, se busca extender sus resultados a la aplicación y em-

\footnotetext{
${ }^{5}$ Del 19 de noviembre de 2001 hasta el 12 de noviembre de 2018 la calificación crediticia soberana de Argentina estuvo por debajo de D (según la escala de Standard \& Poors), razón por la que, en la mayor parte de la simulación realizada, sus mercados de valores tuvieron la tipificación de "frontera". La definición del término mercado desarrollado, mercado emergente y mercado frontera se establece en el Estándar Global de Clasificación de insustrias o Global Industry Classification Standard (GICS). Este fue desarrollado, de manera conjunta, por Morgan Stanley Capital International (MSCI) y Standard \& Poors Dow Jones (S\&P). Esto para tener índices de mercado con una clasificación agregada por países, sectores y tipos de valor. Para mayor referencia de la tipología comentada y de los criterios de clasificación empleados, favor de referirse a MSCI Inc. (2018).
} 
pleo de modelos MS-ARCH y MS-GARCH en la región. Esto en lo que se refiere a su aplicación en la toma de decisiones de inversión.

4. Nada se ha revisado ni escrito respecto al empleo de modelos MS, MS-ARCH o MSGARCH en el proceso de toma de decisiones de inversión en mercados de valores clasificados como "mercados frontera". Esto por parte de Morgan Stanley Capital International (MSCI Inc., 2018), siendo la Bolsa de Valores de Buenos Aires (Argentina) un ejemplo muy representativo de este tipo de mercados y uno muy importante en la actividad económica y financiera de Latinoamérica.

La razón fundamental de elegir estos dos países es el hecho de que estas dos economías son de las más grandes (en términos de su PIB) en Latinoamérica (World Bank, 2019). Por otro lado, Argentina estaba clasificada como un país frontera en la mayor parte del periodo simulado, mientras que México es considerado un país emergente. Por otro lado, el fundamento de realizar una comparación entre los mercados accionarios argentino y mexicano se debe a que el primero, como se dijo previamente, es el más líquido y más grande de los mercados considerados frontera en Latinoamérica y México tiene la divisa más operada en términos de cobertura y operaciones "en el momento" o spot de todos los países emergentes (Bank for International Settlements, 2016).

En específico, se busca demostrar para estos dos mercados que, desde la perspectiva de una o un inversionista cuyo portafolio está denominado en dólares de los Estados Unidos (USD), se pueden lograr, tanto para un mercado emergente como uno frontera, rendimientos superiores. Esto en comparación a una estrategia pasiva o del tipo "comprar y mantener. La posición tomada es que esto se logra al utilizar modelos MS-GARCH dentro del proceso de toma de decisiones de inversión.

Para lograr este objetivo se probará la siguiente estrategia de inversión activa de inversiones:

1. Invertir en el índice de mercado del país simulado si la o el inversionista espera estar en el régimen normal o de baja volatilidad $(s=1)$ en $t+1 \mathrm{o}$

2. Invertir en el activo libre de riesgo en Estados Unidos si la o el inversionista espera estar en el régimen $(s=2)$ de alta volatilidad en el citado periodo..

Dada esta estrategia de inversión y las motivaciones previamente descritas, se establecen dos hipótesis de trabajo a demostrar en el presente:

$\mathrm{H}_{1}$ : "Con el empleo de los modelos MS-GARCH en la estrategia de inversión activa se genera, en los mercados accionarios argentino y mexicano, alpha o rendimientos adicionales respecto a una estrategia pasiva del tipo comprar y mantener".

$\mathrm{H}_{2}$ : "Con el empleo de los modelos MS-GARCH en la estrategia de inversión activa se genera, en los mercados accionarios argentino y mexicano, una reducción significativa 
del nivel de exposición al riesgo. Esto comparado respecto a una estrategia pasiva del tipo comprar y mantener".

La razón de realizar la prueba desde la perspectiva de una o un inversionista denominado en dólares de los Estados Unidos es medir el atractivo que el empleo de la estrategia de inversión tiene para inversionistas extranjeros, siendo los clientes institucionales quienes inyectan mayor operatividad y flujo de dinero hacia estos mercados (Refinitiv, 2018a). De manera complementaria, se fundamenta esta posición en el hecho de que el dólar de los Estados Unidos es la moneda más negociada a nivel internacional (Bank for International Settlements, 2016). Dados estos dos hechos, resulta de interés el revisar si resulta atractivo el invertir en estos dos mercados, con la estrategia de inversión propuesta y desde la perspectiva de una o un inversionista en esta moneda.

Partiendo de la motivación previamente descrita y una vez planteadas las hipótesis de trabajo a demostrar en el presente artículo, se define la siguiente estructura en la situación retórica del documento: en la siguiente sección se hará una breve revisión de la literatura. Esto para contextualizar el empleo de los modelos MS-GARCH en el proceso de toma de decisiones de inversión, así como para contextualizar la estrategia propuesta dentro de la Economía Financiera y en la teoría de portafolios. En la tercera sección se hablará, para fines introductorios para las lectoras y los lectores interesados, del modelo MS-GARCH a utilizar en el contexto del proceso de toma de decisiones de inversión y se hará una descripción del pseudocódigo que rigió las simulaciones. En la cuarta sección se presenta una descripción de los datos de entrada, así como los resultados de las pruebas sobre la pertinencia de emplear modelos MS, MS-ARCH o MS-GARCH en el proceso de toma de decisiones de inversión. En la quinta y última sección, se discuten las principales conclusiones, así como las recomendaciones para futuros trabajos de investigación.

\section{Revisión de literatura}

Los modelos Markov-Switching y su relación con la Teoría de Portafolios y con la administración de inversiones

Un resultado de amplio conocimiento de la propuesta de Markowitz $(1959 ; 1952,1956)$ es el hecho de que se puede estimar, tal como se estableció en la introducción, tanto el nivel de rendimiento esperado como el nivel de exposición al riesgo. Estos son dos parámetros que pueden cuantificarse dentro de una función de utilidad por parte de la o el inversionista, sujeta a maximización. Esto generó una ya conocida evolución del proceso de análisis y toma de 
decisiones de inversión, al grado de que la industria financiera ha tenido avances importantes en cuanto a volumen operado, sofisticación en la asesoría al cliente y en la administración de activos. A pesar de este avance teórico-práctico, la propuesta original de Markowitz adolece de algunas limitantes que se han estudiado y superado en múltiples aspectos. La más importante de ellas es que el vector de rendimientos esperados de cada valor objeto de inversión, así como su correspondiente matriz de covarianzas, son parámetros estadísticos que se toman de manera muestral. Esto es, su valor se determina en función de los datos en la citada muestra, siendo su magnitud sujeta a un nivel de incertidumbre. A este respecto y gracias a las propuestas de Sharpe $(1963,1964)$ y a los trabajos de eficiencia informacional de Fama (1965; 1963), se pueden distinguir dos tipos de administración de portafolios. La primera de ellas se le conoce como administración pasiva (Maggin, Tuttle, Pinto, y McLeavey, 2007) y consiste en invertir en un portafolio cuya conformación es idéntica a la de un portafolio de mercado o índice de referencia. La segunda de estas se conoce como administración activa y consiste en invertir los recursos en un portafolio con una asignación de activos (niveles de inversión) diferente a la observada en el índice de mercado. Esto con la finalidad de generar rendimientos extra o superiores a dicho índice (conocidos como "rendimientos adicionales" o alpha).

En relación con el tema de la incertidumbre a la que los parámetros del modelo de selección de portafolios se refieren, múltiples propuestas se han hecho. Algunas de ellas se pueden apreciar en los trabajos de Michaud y Michaud (1989; 2008) y de Jorion (1992) que utilizan técnicas de remuestreo (simulación Monte Carlo o bootstraping) o el empleo de la Estadística bayesiana como es el modelo de Black y Litterman (1992), del cual han surgido múltiples extensiones (Xiao y Valdez, 2015). Estos últimos casos buscan realizar una combinación lineal entre los parámetros no informados del mercado con las expectativas o pronósticos personales de uno o varios analistas, lo que implica una reducción de la incertidumbre en lo que al vector de rendimientos esperados se refiere. Sin embargo, tanto las técnicas de remuestreo como las bayesianas para la administración de portafolios adolecen de una limitante (en su propuesta original): No distinguen el valor que los parámetros pueden tener en diferentes estados de la naturaleza o regímenes como pueden ser un estado o régimen de baja volatilidad ( $S=1)$ y uno de alta volatilidad $(S=2)$ en donde la fluctuación de los rendimientos tiende ser mayor que en el anterior ${ }^{6}$. Partiendo de esto y observando que, de un periodo a otro, el régimen o estado de la naturaleza puede cambiar, se deben emplear parámetros (vectores de medias y matrices de covarianzas) que sean más representativos de los mismos. Derivado de esto, se desarrollaron dos posibles alternativas para incorporar la incertidumbre generada por la presencia de $S$

\footnotetext{
${ }^{6}$ A este estado también se le conoce como de "crisis" en la literatura relacionada. Esto sin discurrir o contravenir, más bien complementar de forma análoga, otras acepciones del término en la literatura Macroeconómica o de la Teoría Econímoca.
} 
regímenes de la naturaleza. La primera de ellas consiste en utilizar mixturas de funciones de probabilidad como puede ser la gaussiana en donde, por medio de técnicas bayesianas como es el algoritmo E-M de Dempster, Laird y Rubin (1977), se estiman los parámetros de localización (como la media, $\mu_{s}$ ) y de dispersión o escala (como la desviación estándar $\sigma_{s}$ ) para cada régimen y se determina una ley de mixtura que lleva a una combinación lineal de los valores de las dos probabilidades de cada régimen:

$$
P\left(\mu_{s}, \sigma_{s}, \pi\right)=\pi \cdot \Phi\left(\mu_{s=1}, \sigma_{s=1}\right)+(1-\pi) \cdot \Phi\left(\mu_{s=2}, \sigma_{s=2}\right)
$$

Algunas de las aplicaciones de los modelos de mixturas gaussianas en la administración de riesgos o la Econometría Financiera se pueden mencionar en los trabajos de Alexander y Lazar (2006), Bawuen, Hafner y Rombouts (2007), Chung (2009), Haas, Mitnik y Paolella (2004), Geweke y Amisano (2011); Nikolaev, Boshnakov y Zimmer (2013) y Bezerra y Albuquerque (2017). Para el caso específico del empleo de los modelos de mixturas gaussianas en la selección óptima de protafolios "a la Markowitz" se pueden citar los trabajos de Buckley, Sanders y Seco (2008), el de Dark (2015) y el de Levy y Kaplanski (2015). En estas tres fuentes, los autores llegaron a la conclusión en común de que la estimación de parámetros con mixturas gaussianas, lleva a una selección más robusta de portafolios y a mejores valores de desempeño. Esto al incorporar la combinación de los dos o parámetros correspondientes a cada régimen o estado de la naturaleza con la combinación lineal dada en (7). Sin embargo, como se puede apreciar en (7), se logra separar el comportamiento de las series de tiempo de los rendimientos en regímenes pero se maneja el supuesto que la posibilidad de encontrarse en determinado régimen es fija a lo largo del tiempo y está dada por el valor de la ley de mixtura (). Dada esta limitación, es que se puede recurrir a los modelos MS o Markov-Switching propuestos por Hamilton $(1989,1994)$, mismos que se revisaron en la introducción, junto con su extensión de interés para el presente con los modelos MS-GARCH.

Dada la naturaleza dinámica de los modelos MS y la posibilidad de inferir las probabilidades suavizadas y de transición para $S$ regímenes, muchas aplicaciones de estos modelos se han estudiado. Ejemplos de las mismas en el modelado de crisis en mercados financieros y sus contagios a otros se encuentran en las publicaciones de Ang y Bekaert (2002b, 2002c), Kritzman, Page y Turkington (2012)inflation, and economic growth. They found that a dynamic process outperformed static asset allocation in backtests, especially for investors who seek to avoid large losses. nvestors have long recognized that economic conditions frequently undergo regime shifts. The economy often oscillates between a steady, low-volatility state characterized by economic growth and a panic-driven, high-volatility state characterized by 
economic contraction. Evidence of such regimes has been documented in market tur-bulence, 1 inflation, 2 and GDP or GNP. 3 Regime shifts present significant challenges for risk management and portfolio construction. For example, Ang and Bekaert (2002, Klein (2013), Areal, Cortez y Silva (2013), Zheng y Zuo (2013), así como Hauptmann et. al. (2014) (entre otros). En estos artículos se estudia el modelado de mercados accionarios desarrollados como son los de Estados Unidos, Reino Unido, Alemania, Francia, Suiza o Japón. En los mismos, las pruebas se hacen con la presencia de dos o tres regímenes de volatilidad.

Enfocando su atención a otro tipo de valores y su relación con otros mercados, se pueden mencionar las publicaciones de Alexander y Kaeck (2007), Castellano y Scacia (2014) y de Ma, Deng y Ho (2018)we investigate the dependency of short term sovereign credit default swap (SCDS. Estos trabajos estudian el comportamiento de swaps de incumplimiento de pago de crédito o credit default swaps (CDS), así como del contagio de su desempeño en regímenes hacia los de otros mercados como son los accionarios, los cambiarios o los del petróleo.

Para mencionar algunos trabajos que estudian aplicaciones en mercados financieros de países emergentes, se pueden mencionar las publicaciones de Zhao (2010), Walid et. al. (2011), Walid y Duc Khuong (2014), de Rotta and Valls-Pereira (2016), Mouratidis et. al. (2013), Miles y Vijverberg (2011); la de Lopes y Nunes (2012), Kanas (2005), Álvarez-Plata and Schrooten (2006), Parikakis and Merika (2009), Girdzijauskas (2009), Dubinskas and Stungurienè (2010); Kutty (2010), Dufrénot, Mignon, Péguin-Feissolle (2011) y Ahmed et. al. (2018). Todas estas publicaciones citadas se enfocan a estudiar el modelado con $\mathrm{S}$ regímenes, así como el contagio de estos entre mercados accionarios y crediticios emergentes. También revisan la influencia que tiene la política monetaria en el cambio de régimen de paridades cambiarias o en los mercados accionarios.

Algunos de los estudios más representativos en relación al empleo de parámetros MS (vectores de medias y matrices de covarianzas con cambio de régimen) se aprecian en los trabajos de Ang y Bekaert (2002a, 2004), Ishijima y Uchida (2011) y Kritzman, Page y Turkington (2012), quienes sugieren la inferencia de $S$ matrices de covarianzas y vectores de medias. Esto a fin de realizar la selección óptima según las propuestas de selección óptima y racional de Markowitz. Sin embargo, dada la naturaleza computacional de esta estimación y enfocando la inversión a un solo tipo de activo, algunas alternativas pueden plantearse.

Retomando las propuestas del Teorema de la Separación de Fondos de Tobin (1958), así como de los desarrollos de Sharpe $(1963,1964)$, la elección de la o el inversionista se puede reducir a la selección de dos tipos de activos: uno libre de riesgo que, por lo general es el instrumento de mercado de dinero de menor plazo (como la letra del tesoro de los Estados Unidos con 3 meses de vencimiento), así como un activo riesgoso, como puede ser un porta- 
folio teórico que replique el comportamiento de un índice o cartera de mercado. Derivado de esto, se puede reducir el problema de $n$ dimensiones (que implica el elegir óptimamente el nivel de inversión en $n$ activos dentro del portafolio) a uno que se reduzca a cuánto invertir en el activo riesgoso y cuánto en el libre de riesgo (un problema unidimensional). Incluso, el mismo problema se puede simplificar aún más a el caso en el que se decida invertir la totalidad de recursos en el activo riesgoso o en el libre de riesgo si se tiene algún algoritmo o proceso de toma de decisiones que lo permita (como el propuesto en el presente).

Ante esta última idea y empleando los modelos MS, Brooks y Persand (2001) realizan una primera propuesta en la que determinan ya sea invertir en el Gilt o bono de 10 años del Reino Unido o en el índice accionario FTSE100. Esto dada la probabilidad de que la razón tasa gilt/tasa de dividendo FTSE100 se encuentre en el régimen de alta volatilidad o no. Los resultados de estos autores (que no incorporan el impacto de costos financieros) sugieren que el empleo de esta estrategia de administración activa lleva a mejores resultados de desempeño que una estrategia pasiva o de "comprar y mantener" ya sea en el FTSE-100 o en el bono de 10 años británico. De manea análoga se encuentra el trabajo de Hauptman et. al. (2014), quienes desarrollan un sistema de advertencia para el índice $S \&$ P500 para un proceso estocástico con 3 regímenes (baja volatilidad, alta volatilidad con tendencia alcista y alta volatilidad con tendencia bajista). La propuesta de los autores se enfoca a estimar de manera secuencial el modelo de 3 regímenes (para lograr un resultado factible) y a inferir las probabilidades $\xi_{s=i, t}$ de estar en determinado régimen incorporando algunos factores exógenos. Sus resultados (que tampoco incorporan el impacto de costos de transacción) sugieren que el empleo de una estrategia activa con modelos MS lleva a mejores resultados que una estrategia pasiva o de tipo "comprar y mantener".

Por último, se tiene el trabajo de De la Torre, Galeana and Álvarez-García (2018)following Brooks and Persand (2001. Estos autores estudian, de manera explícita, la aplicación de modelos MS en inversiones en mercados accionarios tanto desarrollados (Estados Unidos, Italia y Reino Unido) así como en uno emergente (México). Al incorporar el impacto de un costo de transacción de $0.35 \%$ más impuesto al valor agregado, los autores demuestran que, en los tres casos, el empleo de una estrategia de administración activa (que es la misma que se estudia en el presente artículo) lleva a mejores resultados, en comparación a una pasiva.

Dado lo anterior, se observa, tanto en la revisión de literatura como en la introducción que los trabajos previos que estudian el uso de modelos MS para la toma de decisiones de inversión, que los trabajos previos se enfocan solamente en el empleo de modelos MS con varianza constante y prácticamente todos se enfocan a la aplicación de estos en mercados 
accionarios desarrollados. De todos estos, solo uno se enfoca a uno emergente y ninguno al estudio de los beneficios de estas estrategias en mercados frontera o en mercados de la región latinoamericana. Como resultado de esta necesidad, en términos de heurística positiva para la Economía financiera y para el empleo de modelos MS y MS-GARCH en la toma de decisiones de inversión, es que se realizarán los trabajos del presente artículo para contribuir en la literatura, enfocándonos a los casos particulares de México y Argentina. Esto porque, como se mencionó previamente, son países latinoamericanos y uno de ellos, México, es considerado emergente (con la divisa más líquida de las economías emergentes en el mundo) y el otro es la tercer mayor economía de la región y ha sido considerado un mercado frontero en prácticamente todo el periodo simulado. Dado esto, el comparativo de estos dos países, dadas sus características y su pertenencia a la región, resulta de interés para contribuir a los resultados publicados en el empleo no solo de modelos MS, sino MS-GARCH.

Ya que se fundamentó teóricamente la necesidad del presente, ahora se procederá a dar una breve contextualización del empleo del modelo MS-GARCH para fines de toma de decisiones de inversión.

\section{Metodología de las simulaciones realizadas}

\section{El modelo MS-GARCH y su empleo en la estrategia de inversión activa de inversiones}

El modelo MS-GARCH que se utilizará en las simulaciones del presente trabajo es el que tiene la forma funcional dada en (6). Tal como Haas, Mitnik y Paolella (2004) observan, el modelo MS-GARCH debe ser estimado una vez que los residuales se determinaron de una media aritmética o algún modelo de media condicional, como (1), aplicado en $r_{t}$. Partiendo del hecho de que el presente es una de las primeras aplicaciones de los modelos MS-GARCH en inversiones en mercados frontera, se tomará el supuesto de que el modelo adecuado para determinar el rendimiento esperado o medida de localización será la media aritmética $\mu$ de $r_{t}^{7}$. Lo anterior llevará a $\varepsilon_{t}=r_{t}-\mu$. Estos residuales se utilizarán para inferir los modelos MS, MS-ARCH o MS-GARCH, bajo el supuesto de que el proceso generador o estocástico de la serie de tiempo de los índices es uno con dos regímenes homogéneos (ya sean gaussianos

\footnotetext{
${ }^{7}$ La razón de utilizar los residuales como método de estimación radica en las propuestas de Haas, Mitnik y Paolella (2004). Esto debido a que es más sencilla la estimación del modelo, dada la naturaleza dependiente en el tiempo de las varianzas GARCH en el tiempo (el cual se interrumpe ante un cambio de régimen). De manera complementaria, se utilizará la media como medida de tendencia central, ya que este es el primero de futuros trabajos en la materia.
} 
o t-Student distribuidos). Dado esto, el modelo permitirá también inferir las probabilidades filtradas y sus correspondientes probabilidades suavizadas $\left(\xi_{s, t}\right)$ de estar en el régimen en $t$.

Las funciones de probabilidad para obtener las probabilidades filtradas se dan por las siguientes expresiones para el caso gaussiano y t-Student respectivamente ${ }^{8}$ :

$$
\begin{gathered}
\xi_{s, i, t}=\frac{1}{\sqrt{2 \pi} \sigma_{i, s}} e^{-\frac{1}{2}\left(\frac{\varepsilon_{i, t}}{\sigma_{i, s}}\right)^{2}} \\
\xi_{s, i, t}=\frac{\Gamma\left(\frac{v_{i, s}+1}{2}\right)}{\sqrt{\left(v_{i, s}-2\right) \pi} \Gamma\left(\frac{v_{i, s}}{2}\right)}\left(1+\frac{\left(\frac{\varepsilon_{i, t}}{\sigma_{s}}\right)^{2}}{\left(v_{i, s}-2\right)}\right)^{-\frac{v_{i, s}+1}{2}}
\end{gathered}
$$

En las expresiones anteriores, $v_{s}$ representan los grados de libertad de la distribución t-Student. Estas funciones de probabilidad filtrada permitirán inferir las probabilidades suavizadas $^{9}$, y también llevan a las probabilidades estables (o leyes de mezcla o mixtura) $\pi_{s}$ de la siguiente función de log-verosimilitud a maximizar ${ }^{10}$ :

$$
L\left(r_{i, t}, \theta\right)=\sum_{t}^{T} \ln \left(\sum_{S=1}^{S} \pi_{s} \cdot \xi_{s, i, t}\right), \theta=\left[\sigma_{i, s}, \pi_{s}, \mathbf{\Pi}\right]
$$

Para estimar el vector $\theta$ en (10), se utilizará un método bayesiano de máxima verosimilitud con el algoritmo de Viterbi (1967). De los parámetros estimados con (8) a (10) se prestó interés en las probabilidades suavizadas $\xi_{s, t}$ en $t$, así como en la matriz de probabilidades de transición П. Con estos parámetros se puede pronosticar la probabilidad suavizada de estar en cada régimen en $t+1$. Esto tal como se muestra a continuación:

$$
\left[\begin{array}{l}
\xi_{s=1, t+1} \\
\xi_{s=2, t+1}
\end{array}\right]=\Pi\left[\begin{array}{l}
\xi_{s=1, t} \\
\xi_{s=2, t}
\end{array}\right]
$$

Dadas estas dos probabilidades filtradas, centrando ahora la atención en $\xi_{s=2, t+1}$ y siguiendo lo establecido en la literatura de series de tiempo (Ang y Bekaert, 2002b; Brooks y Persand, 2001; Hamilton, 1989, 1990, 1994; Hauptmann et al., 2014; Kritzman et al., 2012),

\footnotetext{
${ }^{8}$ Estimadas de las probabilidades filtradas según el método de Kim (1994).

${ }^{9}$ Estimadas de las probabilidades filtradas según el método de Kim (1994)

${ }^{10}$ Favor de referirse a Hamilton $(1989,1994)$ para mayor detalle en el desarrollo y la lógica del método de inverencia.
} 
la o el inversionista puede definir si se encontrará en un régimen de alta o baja volatilidad al utilizar la siguiente función indicadora:

$$
s_{t+1}=\left\{\begin{array}{l}
1 \text { si } \xi_{s=2, t+1} \leq 0.5 \\
2 \text { si } \xi_{s=2, t+1}>0.5
\end{array}\right.
$$

Una vez explicado el modelo MS-GARCH y los parámetros a utilizar en la estrategia de inversión a simular, se procederá a una descripción del pseudocódigo que ha regido las simulaciones realizadas.

El pseudocódigo de la estrategia de inversión ejecutado en las simulaciones

Para poder realizar las simulaciones que probarán la utilidad del proceso de toma de decisiones de inversión o estrategia de inversión con modelos MS-GARCH, se supondrá que la o el inversionista tiene un portafolio con un balance inicial de USD 100,000.00 en el cual solo se podrá invertir en dos tipos de activos:

1. El valor base 100, al 7 de junio del año 2000, del índice MSCI Argentina. Este índice será considerado como el precio teórico de un fondo comerciado en bolsa (ETF o Exchange Traded Fund) con un error de réplica o tracking error de cero, hacia el índice MSCI del país simulado.

2. El valor base 100 , también al 7 de junio del año 2000, de un fondo teórico que paga la tasa de rendimiento de la nota del tesoro con 3 meses de vencimiento de los Estados Unidos (USTBILL). Este será considerado como un fondo de inversiones que también no tiene error de réplica o tracking error.

En la Tabla 1 se presenta el resumen de los índices accionarios utilizados como datos de entrada, así como los ticker (o identificadores empelados en el presente artículo) y la definición del tipo de activo que fueron en las simulaciones.

Tabla 1

Los índices accionarios y el activo libre de riesgo de los países andinos que se emplearán en las simulaciones

\begin{tabular}{llllll}
\hline $\begin{array}{l}\text { RIC® de } \\
\text { Refinitiv }\end{array}$ & Fuente de los datos & Nombre del índice & $\begin{array}{l}\text { Ticker utilizado } \\
\text { en el artículo }\end{array}$ & País & $\begin{array}{l}\text { Tipo de activo en las } \\
\text { simulaciones }\end{array}$ \\
\hline $\begin{array}{l}\text { dMIAR- } \\
\text { 00000PUS }\end{array}$ & Refinitiv $^{\mathrm{TM}}$ Eikon $^{\mathrm{TM}}$ & $\begin{array}{l}\text { Índice MSCI } \\
\text { ARGENTINA (USD) }\end{array}$ & MSCIARGUSD & Argentina & $\begin{array}{l}\text { Activo riesgoso (ETF } \\
\text { teórico) }\end{array}$ \\
$\begin{array}{l}\text { dMIMX- } \\
\text { 00000PUS }\end{array}$ & Refinitiv $^{\mathrm{TM}}$ Eikon $^{\mathrm{TM}}$ & $\begin{array}{l}\text { Índice MSCI Mexico } \\
\text { (USD) }\end{array}$ & MSCIMEXUSD & México & $\begin{array}{l}\text { Activo riesgoso (ETF } \\
\text { teórico) }\end{array}$ \\
$\begin{array}{l}\text { UST3M- } \\
\text { T=RR }\end{array}$ & Refinitiv $^{\mathrm{TM}}$ Eikon $^{\mathrm{TM}}$ & $\begin{array}{l}\text { Nota del tesoro de los } \\
\text { EUA con } 3 \text { meses de } \\
\text { vencimiento. }\end{array}$ & USTBILL & $\begin{array}{l}\text { Estados } \\
\text { Unidos }\end{array}$ & $\begin{array}{l}\text { Activo libre de riesgo } \\
\text { (fondo teórico) }\end{array}$ \\
\hline
\end{tabular}

Fuente: Elaboración propia. 
La razón de utilizar la familia de índices MSCI se fundamenta en el hecho de que la misma es una ampliamente reconocida y empleada para fines de benchmarking o referenciación en el diseño de la política de inversión de portafolios internacionalmente diversificados (Bodie, Kane, y Marcus, 2014; Maggin et al., 2007).

Los datos de la series de tiempo de los precios de los índices, así como la tasa de los USTBILL fueron extraídos de las bases de datos Refinitiv ${ }^{\mathrm{TM}}$ Eikon $^{\mathrm{TM}}$ (Refinitiv, 2018b) y fueron transformadas por el método de rendimientos continuamente capitalizables dado en (2). Con esto, se determinó una serie de tiempo de rendimientos $\left(r_{t}\right)$ con 1,078 observaciones (desde enero de 1998). Las tres series de tiempo comienzan el 6 de junio de 1998 y terminan el 31 de enero de 2019 (1,079 observaciones). Como se mencionará posteriormente, las simulaciones iniciarán desde el primero de enero del 2000 hasta el 30 de enero de 2019 (996 semanas de simulación) y los históricos de los rendimientos $\left(r_{t}\right)$ de las semanas previas desde junio de 1998 hasta $t$, se utilizarán para fines de estimación de los parámetros de los modelos MS-GARCH.

Dado que esta prueba es una primera de varias que se pretenden realizar para los mercados accionarios frontera y emergentes latinoamericanos, y en línea con la mayoría de las publicaciones previas relacionadas y descritas en la sección de revisión de literatura, no se considerará el pago de costos de corretaje ni impuestos en las operaciones de compra y venta en el índice. De manera complementaria, se dejará de lado el impacto del riesgo cambiario ${ }^{11}$, así como impactos de mercado por la fluctuación de precios al realizar las operaciones de compra o venta (slipage).

Por otro lado, el portafolio simulado comprendió de solo dos subcuentas:

1. Una subcuenta de custodia de valores en donde se encontrará el valor de mercado de las inversiones realizadas en los activos previamente descritos y

2. Una subcuenta de efectivo en donde se depositarán los montos residuales que, dado el número de títulos y el precio de los activos objeto de inversión, quedaron a la vista.

Con estos supuestos y parámetros, se simulará (en las 996 semanas del periodo simulado) la ejecución recursiva del siguiente pseudocódigo:

Ciclo, de la semana $t=1$ a 996 :

1. Cuantificar el balance actual del portafolio al sumar el balance de la subcuenta de efectivo a la vista más el balance del valor de mercado de los activos que se encuentran en la subcuenta de custodia.

${ }^{11} \mathrm{Al}$ estar calculados, de origen, los índices de la familia MSCI en dólares de los Estados Unidos. 
2. Ejecutar el análisis con el modelo MS (MS con varianza constante, MS-ARCH o MS-GARCH) dado en (6). Esto ya sea con función de verosimilitud gaussiana y/o t-Student homogénea en los dos regímenes.

3. Estimar la probabilidad suavizada $\left(\xi_{s=2, t+1}\right)$ del régimen de alta volatilidad para $t+1$ con (11).

4. Si $s_{t+1}=2\left(\xi_{s=2, t+1}>0.5\right)$, entonces:

a. Invertir en el fondo que paga la tasa de los USTBILL (Treasury-Bill ETF). Si no:

b. Invertir en el activo riesgoso (El ETF que replica el comportamiento del índice simulado).

5. Valuar el portafolio a precios de Mercado (mark-to-market) con los precios de cierre en $t$.

\section{Finalizar ciclo}

Los resultados logrados tanto para la estrategia de inversión pasiva (comprar y mantener) en los tres índices simulados, así como los resultados de la administración activa realizada con el pseudocódigo anterior se presentan en la siguiente sección.

\section{Discusión de los resultados de las simulaciones}

\section{Descripción estadística de los datos a utilizar en las simulaciones}

El resumen estadístico de las series de tiempo de los rendimientos históricos de los índices accionario y de la tasa equivalente semanal del activo libre de riesgo se exponen en la Tabla 2. Como se aprecia en la misma, el promedio de rendimiento medio semanal pagado por el MSCI rgentina es de $0.20 \%$, que es mayor al $0.0373 \%$ semanal que recibiría la misma inversionista si hubiese invertido su dinero en las notas del Tesoro de los Estados Unidos ${ }^{12}$. De manera análoga, se puede apreciar prácticamente el mismo resultado para el caso mexicano con un rendimiento medio de $0.21 \%$. Un resultado que sí logra presentar un factor diferenciador entre Argentina y México es el rango que se da con los valores mínimo y máximo. En estos, el índice argentino presenta magnitudes más extremas. Esto, claro está, aplica para todo el periodo revisado desde junio de 1998 hasta enero de 2019.

\footnotetext{
${ }^{12}$ Que es, en la práctica profesional, considerado el activo libre de riesgo para una o un inversionista basado en dólares de los Estados Unidos -USD-).
} 
Tabla 2

Resumen estadístico de los rendimientos semanales de los índices accionarios y active libre de riesgo estudiados en el presente artículo (valores en \%)

\begin{tabular}{lllllll}
\hline Ticker & Mínimo & Cuantil 5\% & Media & $\begin{array}{l}\text { Desviación } \\
\text { estándar }\end{array}$ & Cuantil 95\% & Máximo \\
MSCIARGUSD & -28.5700 & -8.1700 & 0.2000 & 5.4900 & 8.3100 & 30.3600 \\
MSCIMEXUSD & -26.4200 & -6.2000 & 0.2100 & 4.0100 & 5.8900 & 25.3000 \\
USTBILL & -0.000254 & 0.000304 & 0.037354 & 0.039262 & 0.10538 & 0.12754 \\
\hline
\end{tabular}

Fuente: Elaboración propia con datos de Thomson Reuters (2018b).

Para determinar si los modelos MS, MS-ARCH o MS-GARCH son apropiados para aproximar el comportamiento del proceso estocástico de la serie de tiempo de los residuales de los índices accionarios, se calculó la función de verosimilitud gaussiana y t-Student. Esto considerando la presencia de un solo régimen. Posterior a ello, se aplicó el filtro de Hamilton $(1989,1994)$ para inferir los parámetros de los modelos MS, MS-ARCH y MS-GARCH (gaussianos y t-Student ${ }^{13}$ ).

Tabla 3

Resumen de los valores de criterio de información bayesiana para todos los modelos Ms estudiados en cada índice accionario de interés

\begin{tabular}{lll}
\hline Proceso estocástico & MSCIARGUSD & MSCIMEXUSD \\
\hline Gaussiano (un régimen) & $-3,181.83$ & $-3,858.55$ \\
t-Student (un régimen) & $-3,349.74$ & $-4,038.77$ \\
MS-Gaussiano & $-3,364.2598[*]$ & $-4,093.52$ \\
MS-tStudent & $-3,359.65$ & $-4,098.02$ \\
MSARCH-Gaussiano & $-3,350.29$ & $-4,092.63$ \\
MSARCH-tStudent & $-3,347.21$ & $-4,093.82$ \\
MSGARCH-Gaussiano & $-3,360.78$ & $-4,108.14[*]$ \\
MSGARCH-tStudent & $-3,354.12$ & $-4,102.71$ \\
\hline
\end{tabular}

Fuente: Elaboración propia con datos de Thomson Reuters (2018b).

Una vez realizado esto, se calculó el Criterio de información de Akaike (1974)often multisensory events that rely on binding different elements [1]. The elements that will constitute a memory episode are processed in specialized but distinct brain modules. The binding of these elements is most likely mediated by fast-acting long-term potentiation (LTP. Esto se hizo así

\footnotetext{
${ }^{13}$ Función que es homogénea o la misma para los dos regímenes.
} 
porque solo se manejará un rezago tanto en el término ARCH como GARCH en el modelo inferido ${ }^{14} \mathrm{y}$, dado que el número de parámetros es fijo, no se ve de necesidad utilizar algún estadístico que haga un equilibrio entre el número de parámetros (parsimonia del modelo) y la precisión en el modelo estimado. Debido a que se busca, con el número de parámetros fijo, buscar el modelo que sea más preciso para en su ajuste a la serie de tiempo, se tomó la determinación de utilizar el AIC. Dicho esto, se presenta, en la Tabla 3, el comparativo de los valores del AIC calculado para las series de tiempo de rendimientos en ambos índices accionarios.

Como se puede apreciar, resulta más apropiado utilizar un proceso estocástico de dos regímenes para modelar el comportamiento de la serie de tiempo de rendimientos $\left(r_{t}\right)$ de los índices. De manera más precisa, dentro de los 6 procesos estocásticos estudiados en este trabajo, el modelo MS gaussiano con varianza constante se aprecia como el mejor para modelar el comportamiento del índice MSCI argentino y el MS-GARCH gaussiano el del mexicano.

Dicho esto, ahora se procederá a la revisión de los resultados de las simulaciones realizadas, en donde se estimaron los seis modelos MS propuestos en cada semana.

\section{Discusión e interpretación de los resultados observados en las simulaciones}

Como primer punto de partida, se presenta, en la Tabla 4, el resumen de desempeño de la estrategia de inversión pasiva (comprar y mantener) realizada ya sea en los ETF teóricos de los índices simulados o en el fondo que paga la tasa de los USTBILL. Como se puede apreciar e independientemente del rendimiento medio semanal plasmado en la Tabla 2, el invertir en el mercado accionario argentino le permitió a la o el inversionista lograr un rendimiento acumulado de 56.1372\% (2.0040\% anual), el cual es superior al 38.38\% (2.9308 anual) que le hubiese pagado el invertir su dinero en los USTBILL. De manera análoga, se puede apreciar el rendimiento acumulado de $162.06 \%$ (8.92\% anual) que la o el inversionista hubiese logrado en el MSCI México.

Para el periodo observado y dada la presencia de una crisis financiera y fiscal en Argentina en la parte inicial del periodo de estudio, se aprecia que el MSCI Argentina, tuvo un rendimiento acumulado y medio superior a los USTBILL, pero de prácticamente un tercio del rendimiento acumulado o casi la mitad del rendimiento medio logrado en el caso mexicano.

\footnotetext{
${ }^{14}$ La librería MSGARCH empleada en este trabajo no permite calcular modelos ARCH y GARCH con más de un rezago en sus términos. Es una extensión en la que se busca trabajar en el futuro.
} 
Tabla 4

Resumen del desempeño de la administración pasiva realizada en cada índice y el activo libre de riesgo objeto de estudio (datos en porcentaje excepto índice de Sharpe)

\begin{tabular}{lllll}
\hline Ticker & Rendimiento acumulado & Rendimiento medio & $\begin{array}{l}\text { Desv. Est. de } \\
\text { rendimiento }\end{array}$ & $\begin{array}{l}\text { Peor desempeño } \\
\text { semanal }\end{array}$ \\
\hline MSCIARGUSD & $56.1372[2.9308]$ & 0.0448 & 5.5518 & -33.6472 \\
MSCIMEXUSD & $162.0699[8.9213]$ & 0.0968 & 3.8617 & -30.6773 \\
USTBILL & $38.3854[2.0040]$ & 0.0374 & 0.0393 & - -- \\
\hline
\end{tabular}

Fuente: Cálculos propios en base a las simulaciones realizadas con datos de Thomson Reuters (2018b).

De interés adicional son los resultados de exposición al riesgo. Tanto las cifras de Valor en Riesgo condicional $(\mathrm{CVaR})^{15}$ como el peor desempeño semanal (conocido también como max drawdown ${ }^{16}$ ). Los valores para ambos índices accionarios son altos y muy aproximados. Partiendo de este resultado, es que se tiene sustento para buscar demostrar la hipótesis de que, adicional a una mejora en el rendimiento acumulado, se puede reducir significativamente el nivel de exposición al riesgo si se realiza administración activa con el sistema de inversión propuesto en el presente.

Dado esto, la idea básica de la administración activa de inversiones es reducir, por un lado, estas pérdidas potenciales, así como incrementar los rendimientos logrados por medio de operaciones más periódicas de compra y venta. Con esta premisa se tiene la expectativa de lograr estos dos objetivos, al ejecutar la estrategia de inversión sugerida en el presente artículo, cuyo pseudocódigo se describió en la sección anterior.

\footnotetext{
${ }^{15}$ Calculado con el siguiente método: $C V a R=\int_{-\infty}^{\alpha} r_{i} p\left(r_{i}\right) d r_{i}, \quad r_{i} \leq \alpha, \alpha=\operatorname{VaR}\left(r_{i}\right)$ a $95 \%$ de confianza.

${ }^{16}$ Calculado como .(( $\left.\left(r_{i, t}\right)\right)$
} 


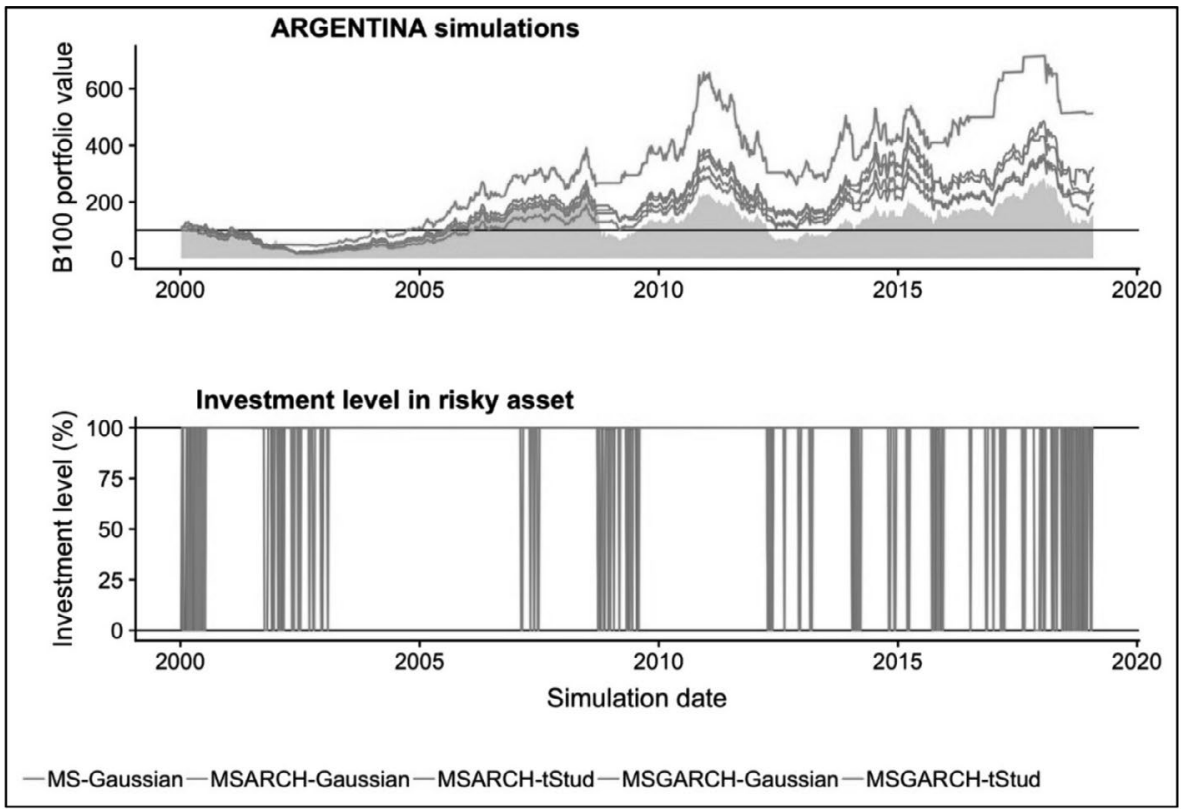

Figura 1. Desempeño histórico de los portafolios simulados en el mercado argentino.

Fuente: Cálculos propios en base a las simulaciones realizadas con datos de Thomson Reuters (2018b).

\section{Tabla 5}

Resumen del desempeño de la administración activa, desde la perspectiva de un inversionista con un portafolio denominado en dólares de Estados Unidos, al invertir en el índice MSCI Argentina con modelos Markov-Switching

Resultados observados en el mercado argentino

\begin{tabular}{|c|c|c|c|c|c|}
\hline $\begin{array}{l}\text { Modelo } \\
\text { Markov-Switching } \\
\text { utilizado }\end{array}$ & $\begin{array}{l}\text { Rendimiento } \\
\text { acumulado }\end{array}$ & $\begin{array}{l}\text { CVaR } \\
(98 \%)\end{array}$ & $\begin{array}{l}\text { Peor } \\
\text { desempeño } \\
\text { semanal }\end{array}$ & $\begin{array}{l}\text { índice de } \\
\text { Sharpe }\end{array}$ & $\begin{array}{l}\text { Nivel medio de } \\
\text { inversión en activo } \\
\text { riesgoso }\end{array}$ \\
\hline MS-Gaussiano & $\begin{array}{l}100.0754 \\
{[5.2248]}\end{array}$ & -12.0474 & -14.9487 & -0.0041 & 96.15 \\
\hline MS-tStudent & No factible & No factible & No factible & No factible & No factible \\
\hline $\begin{array}{l}\text { MSARCH- } \\
\text { Gaussiano }\end{array}$ & $\begin{array}{l}164.3827 \\
{[8.5822]}\end{array}$ & -12.0135 & -14.9517 & 0.0017 & 95.15 \\
\hline $\begin{array}{l}\text { MSARCH- } \\
\text { tStudent }\end{array}$ & $\begin{array}{l}143.581 \\
{[7.4962]}\end{array}$ & -12.4817 & -19.4665 & -0.0049 & 95.53 \\
\hline $\begin{array}{l}\text { MSGARCH- } \\
\text { Gaussiano }\end{array}$ & $\begin{array}{l}223.8074 \\
{[11.6847]}\end{array}$ & -11.9985 & -14.9551 & 0.0082 & 92.74 \\
\hline $\begin{array}{l}\text { MSGARCH- } \\
\text { tStudent }\end{array}$ & $\begin{array}{l}412.8318 \\
{[21.5535]}\end{array}$ & -11.5586 & -19.4741 & 0.0162 & 92.05 \\
\hline
\end{tabular}


Resultados observados en el mercado mexicano

\begin{tabular}{llllll}
\hline $\begin{array}{l}\text { Modelo } \\
\text { Markov-Switching } \\
\text { utilizado }\end{array}$ & $\begin{array}{l}\text { Rendimiento } \\
\text { acumulado }\end{array}$ & $\begin{array}{l}\text { CVaR } \\
(98 \%)\end{array}$ & $\begin{array}{l}\text { Peor desem- } \\
\text { peño semanal }\end{array}$ & $\begin{array}{l}\text { índice de } \\
\text { Sharpe }\end{array}$ & $\begin{array}{l}\text { Nivel medio de } \\
\text { inversión en activo } \\
\text { riesgoso }\end{array}$ \\
\hline MS-Gaussiano & $\begin{array}{l}259.3627 \\
{[13.541]}\end{array}$ & -8.3678 & -17.4144 & 0.0147 & 0.9676 \\
MS-tStudent & $\begin{array}{l}255.8699 \\
{[13.3587]}\end{array}$ & -7.9479 & -10.1805 & 0.0223 & 0.9636 \\
$\begin{array}{l}\text { MSARCH- } \\
\text { Gaussiano }\end{array}$ & $\begin{array}{l}75.7788 \\
{[3.9563]}\end{array}$ & -7.8676 & -10.1763 & -0.0048 & 0.9493 \\
MSARCH-tStudent & $\begin{array}{l}95.3431 \\
{[4.9778]}\end{array}$ & -8.8926 & -17.4112 & 0.0034 & 0.9713 \\
MSGARCH- & 154.7175 & -7.8395 & -10.1853 & 0.011 & 0.9172 \\
$\begin{array}{l}\text { Gaussiano } \\
\text { MSGARCH- }\end{array}$ & $\begin{array}{l}{[8.0776]} \\
\text { tStudent }\end{array}$ & & & & \\
\hline
\end{tabular}

Fuente: Elaboración propia con datos de las simulaciones realizados y de Thomson Reuters (2018b).

Para verificar esto y después de realizar las simulaciones de eventos previamente descritas, se presentan los resultados observados para el caso argentino. Esto en la parte superior de la Tabla 5 (los valores de todos los campos, excepto índice de Sharpe, se presentan en porcentaje). Como se aprecia en la misma, el utilizar modelos MS-GARCH t-Student lleva al mejor desempeño, al lograrse un rendimiento acumulado de $412.8318 \%$ (21.5535\% anual). Le siguen en resultados logrados el empleo del modelo MS-GARCH gaussiano con 223.8074\% (11.6847\%), el MS-ARCH gaussiano con 164.3827\% (8.5822\%), el MS-ARCH t-Student distribuido que pagó $143.5810 \%(7.4962 \%)$ y el MS (con varianza constante) gaussiano que permitió obtener un 100.0754\% (5.2248\%) de rendimiento. Para el caso específico del modelo MS con varianza constante y función de verosimilitud t-Student se observa la leyenda "no factible". Esto implica que, en algunas de las semanas de simulación"17, el método de estimación no llevó a una convergencia en el problema de máxima verosimilitud empleado y, por tanto, no se tenían valores de la probabilidad de estar en el periodo de alta volatilidad en . Dado esto, se excluyó de la simulación el empleo de este modelo.

A manera de resumen de los resultados de rendimiento acumulado, se puede apreciar que, para el caso argentino se logra el objetivo del presente trabajo y se prueba la hipótesis

\footnotetext{
${ }^{17}$ Las fechas en que no se logró una convergencia en el problema de optimización fueron 6: 2 de febrero de 2001, 14 de septiembre de 2001, 21 de abril de 2006, 06 de octubre de 2006, 03 de noviembre de 2006 y 02 de febrero de 2007. Las razones de esto se deben a cuestiones propias del método de inferencia empleado. Dado esto, se sugiere utilizar métodos alternativos como la simulación Monte Carlo con Cadenas Markovianas (Markov Chain Monte Carlo).
} 
que afirma que se genera un rendimiento adicional a la estrategia pasiva (alpha) si se utiliza la estrategia de inversión propuesta en el presente.

De manera complementaria, se puede apreciar la mejora en el nivel de exposición al riesgo en los portafolios simulados. Por ejemplo, el peor desempeño semanal (max drawdown) observado fue el caso del portafolio con modelos MS-GARCH t-Student que tuvo una caída de $-19.7441 \%$. Esta es notablemente inferior al peor desempeño semanal observado en la estrategia pasiva o de comprar y mantener (Tabla 4) que presentó una caída de $-33.6472 \%$. Con esto, la segunda hipótesis de reducción del nivel de exposición al riesgo se comprueba para el caso argentino.

Con la idea de dar mayor fundamento a la exposición de resultados, se presenta, en la figura 1, el desempeño histórico de los seis portafolios simulados en este mercado (Argentina). En la misma, se puede apreciar el desempeño superior que el escenario que emplea el modelo MS-GARCH t-Student tiene resultados superiores a una estrategia del tipo "comprar y mantener" (su desempeño se expone como un área sombreada). El resultado observado se debe a que su el empleo del modelo MS-GARCH gaussiano llevó a decisiones de inversión más precisas. Esto se puede apreciar en periodos conocidos de alta volatilidad o crisis en los mercados como son octubre de 2008, la crisis de deuda europea de marzo-julio de 2013, julio-noviembre de 2016 y los últimos meses del año 2019. Estos periodos son reconocidos por ser episodios de crisis en los mercados financieros o situaciones de orden político que incrementaron notablemente los niveles de volatilidad. Esto al grado de que fueron identificados como periodos del segundo régimen, llevando a una decisión de desinversión en el mercado accionario. Son precisamente esas tomas de decisión de invertir en el activo libre de riesgo, que permitieron que, durante la caída de los mercados en dicho periodo, el portafolio simulado en este escenario tuvo un comportamiento relativamente plano al no estar expuesto al nivel de riesgo observado en esos momentos. Esto es, el portafolio simulado logró mejores resultados de desempeño gracias a que estaba desinvertido en periodos de baja en generalizada en los precios de las acciones. Dado esto, se logra observar el fundamento del beneficio de emplear estos modelos (MS-GARCH t-Student) para toma de decisiones de inversión en el mercado argentino. 


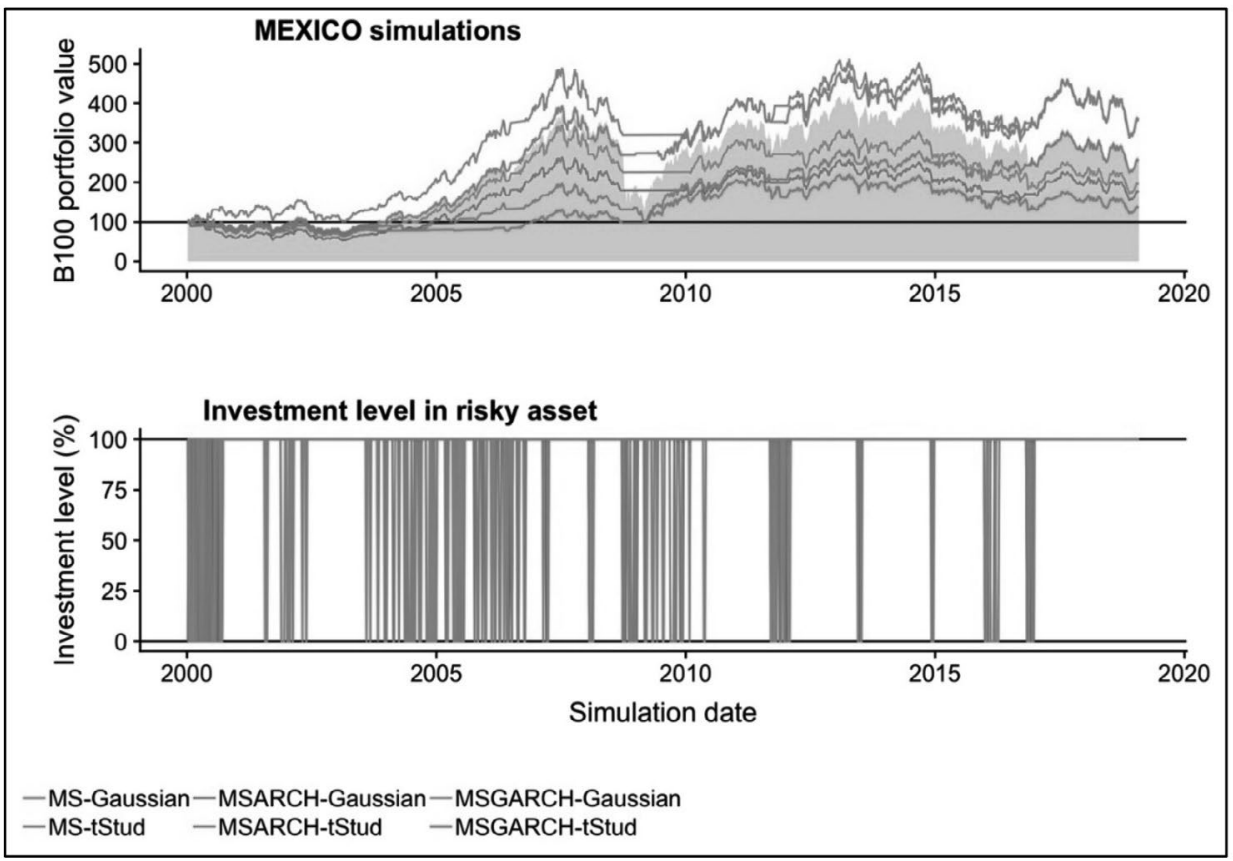

Figura 2. Desempeño histórico de los portafolios simulados en el mercado mexicano.

Fuente: Cálculos propios en base a las simulaciones realizadas con datos de Thomson Reuters (2018b).

Para el caso mexicano, se pueden observar sus resultados en el panel inferior del cuadro 5. Como se puede apreciar, el modelo MS gaussiano con varianza constante es el que lleva a mejores resultados de rendimiento acumulado. Esto con un $259.36 \%$ de rendimiento acumulado ( $13.51 \%$ anualizado), seguido del mismo modelo con función de verosimilitud t-Student que logró un 255.86\% (13.35\%). Como se puede apreciar, en este caso particular también se logra alpha o rendimientos adicionales a los logrados con una estrategia pasiva. Sin embargo, esto se logra con modelos MS de varianza constante. Este resultado es de interés ya que contradice tanto a los resultados de bondad de ajuste de la Tabla 3 como a los observados en el mercado argentino en donde es mejor emplear modelos MS-GARCH. Esto lleva a un resultado de potencial interés y a una futura guía de investigación. También resalta que, en México, un mercado emergente latinoamericano, resultan más adecuados los modelos MS con varianza constante para realizar administración activa y en un mercado considerado "frontera" como el argentino, resulta más atractivo el empleo de modelo MS-GARCH.

En relación con los niveles de exposición al riesgo observados para el portafolio simulado en este mercado, se puede apreciar que, al igual al caso argentino, se cumple la segunda 
hipótesis en la que se afirma que se tiene una observable reducción en la exposición al riesgo si se realiza administración activa de portafolios.

De manera análoga al análisis realizado en el mercado argentino, se presenta la Figura 2. En la misma se expone el desempeño histórico de los portafolios simulados en este país y se aprecia el fundamento de por qué el escenario que emplea el modelo MS t-Student distribuido es el que presenta los mejores resultados de desempeño. En específico, se puede apreciar cómo en el periodo octubre de 2008 a febrero de 2009, este portafolio estuvo desinvertido en el mercado accionario. Este resultado se puede apreciar claramente en el comportamiento de este, el cuál fue de una línea recta. Esto último es resultado de estar invertido en el cativo libre de riesgo. De manera complementaria, el portafolio tuvo niveles de inversión nulos en el mercado accionario durante este periodo, así como en el periodo de la crisis de mediados del 2013. Esto permitió generar diferencias importantes en términos desempeño, en relación con una estrategia del tipo "comprar y mantener" (área sombreada en la gráfica).

Como corolario de los resultados observados, se puede notar que el empleo de modelos MS-GARCH lleva a mejores resultados de desempeño y a decisiones de inversión adecuadas. Estas últimas permiten mejorar el desempeño en relación con una estrategia de inversión pasiva. De manera complementaria y como fundamento de lo anterior, la toma de decisiones lograda con el empleo de estos modelos permite reducir el nivel de exposición al riesgo. En específico, en tiempos de muy alta volatilidad, el modelo permite reducir las posiciones en acciones, de tal manera que los movimientos de baja no se vean afectados.

Con los resultados presentados, se puede apreciar que el empleo de modelos MS-GARCH con función de verosimilitud gaussiana (para el caso argentino) y el MS t-Student (con varianza fija en el tiempo) llevan al mejor desempeño. Quizá, para el caso mexicano, no se logró cumplir a totalidad la hipótesis de trabajo (el mejor desempeño no se logra con un modelo MS-GARCH, sino con uno MS) pero se logró demostrar la pertinencia del empleo de este tipo de modelos para la toma de decisiones. De manera complementaria, el empleo de modelos MS-GARCH permitió que se tuvieran decisiones más adecuadas en un mercado frontera como el argentino. Esto podría llevar a la suposición de que el empleo de modelos MS con varianza constante es adecuado en mercados que no se catalogan como frontera y el empleo de modelos MS-GARCH lo es para mercados frontera o con mayor nivel de exposición al riesgo.

\section{Conclusiones}

Los modelos markovianos de cambio de régimen, Markov-Switching o MS (Hamilton, 1989, $1990,1994)$ fueron propuestos para modelar series de tiempo cuyo comportamiento. Esto 
cuando su comportamiento sea propio de un proceso estocástico con $s \geq 2$ regímenes o estados de la naturaleza. Esto es, que sea un proceso estocástico proveniente no de una, sino de dos o más funciones de probabilidad, mismas que tengan $s \geq 2$ parámetros de localización, dispersión y forma.

Al momento de redactar el presente trabajo, múltiples aplicaciones de este tipo de modelos se han propuesto y estudiado. Ejemplos de estas son la cuantificación de la probabilidad de que determinada economía se encuentre en un periodo de recesión o no. También se ha estudiado el efecto de contagio (conocido como spillovers) de la política monetaria de un banco central a los mercados cambiarios o accionarios.

Otra aplicación poco estudiada de los modelos MS es su empleo en el proceso de toma de decisiones en estrategias de inversión activa. Casos, en específico, de esta aplicación son las propuestas primigenias de Brooks y Persand (2001), Ang y Bekaert (2002a), Kritzman, Page y Turkington (2012), Hauptmann et. al. (2014). También lo son las pruebas realizadas por De la Torre, Galeana y Álvarez-García (2018) que se enfocan al empleo de los modelos MS en la administración activa de índices accionarios de países desarrollados y de México (país emergente). Partiendo de esta revisión, poco se ha estudiado con detalle en la materia (empleo de modelos MS en inversiones) para otras economías emergentes y nada se ha escrito sobre el empleo de modelos MS, MS-ARCH o MS-GARCH tanto en mercados desarrollados como emergentes y mercados frontera. Dada esta carencia y motivación, se realiza el presente análisis en Argentina, considerado un mercado accionario frontera, y en México, un país emergente. Esto desde la perspectiva de una o un inversionista cuya cartera está denominada en dólares de los Estados Unidos.

De manera complementaria, se observó que los trabajos que prueban los modelos de administración activa con modelos MS, solo lo hacen con una función de verosimilitud gaussiana y con varianza convencional o constante a lo largo del tiempo. Dado esto, en el presente se extienden los estudios en la materia, al utilizar modelos MS con varianzas ARCH o GARCH y se contrastaron los resultados de desempeño que se lograrían si una o un inversionista hubiera realizado administración activa de portafolios con los mismos. Lo anterior al utilizar la siguiente estrategia de inversión:

1. Invertir en el índice de mercado del país estudiado si la o el inversionista espera estar en el régimen normal o de baja volatilidad $(S=1) t+1$ en o

2. Invertir en el activo libre de riesgo en Estados Unidos si la o el inversionista espera, para el mismo periodo de tiempo $(t+1)$, estar en el régimen $(S=2)$ de alta volatilidad. Con esta estrategia de inversión se planteó la demostración de dos hipótesis de trabajo que resultan complementarias entre sí: 
$\mathrm{H}_{1}$ : "Con el empleo de los modelos MS-GARCH en la estrategia de inversión activa se genera, en los mercados accionarios argentino y mexicano, alpha o rendimientos adicionales respecto a una estrategia pasiva del tipo comprar y mantener".

$\mathrm{H}_{2}$ : "Con el empleo de los modelos MS-GARCH en la estrategia de inversión activa se genera, en los mercados accionarios argentino y mexicano, una reducción significativa del nivel de exposición al riesgo. Esto comparado respecto a una estrategia pasiva del tipo comprar y mantener".

Para comprobar estas dos hipótesis, se realizaron simulaciones semanales de enero del año 2000 hasta enero del año 2019 (996 semanas). Esto, como se mencionó, en los mercados accionarios argentino y mexicano. De manera complementaria, se infirieron recursivamente, en cada fecha, los modelos MS, MS-ARCH o MS-GARCH. Dado esto, se estimaron las probabilidades suavizadas de estar en el régimen de alta volatilidad en $t+1$ para realizar la estrategia de inversión previamente descrita. En los resultados de las simulaciones se observó que los modelos MS-GARCH con función de verosimilitud t-Student llevan, para el caso del mercado argentino, a rendimientos acumulados de $412.8318 \%$ (21.5535\% anual). Esto comparado con un rendimiento acumulado de $56.1372 \%$ ( $2.9308 \%$ anual) y de $38.3854 \%$ (2.0040\% anual) logrados al invertir pasivamente en el índice MSCI argentina y las letras del Tesoro Estadounidense. De manera complementaria, se aprecia que los niveles de exposición al riesgo se reducen gracias a esta administración activa.

Para el caso específico del mercado accionario mexicano, se observa que el empleo de los modelos MS gaussianos con varianza constante llevan a un $259.36 \%$ de rendimiento acumulado (13.51\% anualizado). Un valor que es notablemente superior al 162.06\% (8.92\% anualizado) observado en la estrategia pasiva de este mercado.

Como se puede apreciar al contrastar un mercado emergente latinoamericano (México) y uno frontera de la misma región (Argentina), se aprecia que las hipótesis de trabajo planteadas en este artículo se cumplen cabalmente para el mercado argentino. Para el caso mexicano, la primera hipótesis de trabajo se cumple parcialmente. Esto es, se genera alpha pero no es por medio de modelos MS-GARCH, sino a través de modelos MS gaussianos con varianza constante, la propuesta original de Hamilton $(1989,1994)$.

Los resultados observados presentan implicaciones de potencial interés para la práctica profesional de la administración de inversiones, ya que el empleo de modelos MS con varianza constante y MS-GARCH permiten generar alpha o rendimientos marginales respecto a una estrategia pasiva y permitirían que una o un inversionista denominada en dólares pueda comprar y vender el MSCI argentina o el MSCI México. Esto, según las expectativas o pro- 
babilidad de estar en un régimen de alta volatilidad en $t+1$. Esta práctica puede revolucionar, por el lado tanto de oferta como de la demanda, al mercado de sociedades de inversión o el propio de los exchange traded funds (ETF) o fondos cotizados en bolsa. Esto gracias a que la selección de dos activos, planteada en esta estrategia de inversión en México (mercado emergente) y Argentina (mercado frontera), permitiría que la o el inversionista pueda invertir activamente en este tipo de mercados de una manera más precisa (en términos de la toma de decisiones de inversión y desinversión) y por medio de un solo vehículo diversificado (el ETF o la sociedad de inversión).

Dentro de las áreas de oportunidad que se identifican para futuros trabajos de investigación y dadas las limitantes del trabajo señaladas en la revisión de resultados, se proponen las siguientes guías:

1. Desarrollar y simular estrategias de inversión con más de dos regímenes.

2. Simular estas estrategias de inversión con modelos MS, MS-ARCH o MS-GARCH con funciones de verosimilitud heterogéneas en cada régimen.

3. Emplear modelos asimétricos en los parámetros de volatilidad y en las funciones de verosimilitud.

4. Incorporar el impacto de costos financieros de transacción, así como otros riesgos de mercado no incorporados aquí como son el slipage (o fluctuación del precio de ejecución) el riesgo cambiario o algún otro riesgo o impacto debido a la influencia de variables exógenas o eventos no incorporados en el modelo MS utilizado.

Con los resultados aquí presentados, se espera lograr una contribución significativa a la literatura del empleo de modelos MS. En específico, al empleo de estos para fines de administración activa de portafolios en mercados de valores clasificados como "mercados frontera" y en "mercados emergentes". De manera complementaria, se espera contribuir al estudio de los beneficios de invertir de manera activa, empleando modelos MS-GARCH.

\section{Referencias}

Ahmed, R. R., Vveinhardt, J., Štreimikiene, D., Ghauri, S. P., y Ashraf, M. (2018). Stock returns, volatility and mean reversion in Emerging and Developed financial markets. Technological and Economic Development of Economy, 24 (3): 1149-1177.

Akaike, H. (1974). A new look at the statistical model identification. IEEE transactions on automatic control, 19 (6): 716-723.

Alexander, C., y Kaeck, A. (2007). Regime dependent determinants of credit default swap spreads. Journal of Banking \& Finance, (32): 1008-1021.

Alexander, C., y Lazar, E. (2006). Normal mixture GARCH(1,1): Applications to exchange rate modelling. Journal of Applied Econometrics, 21 (3): 307-336. 


\section{O. V. De la Torre-Torres / Contaduría y Administración 66(1), 2021, 1-32}

http://dx.doi.org/10.22201/fca.24488410e.2020.2657

Alvarez-Plata, P., y Schrooten, M. (2006). The Argentinean currency crisis: A Markov-switching model estimation. Developing Economies, 44 (1): 79-91.

Ang, A., y Bekaert, G. (2002a). International Asset Allocation With Regime Shifts. The review of financial studies, 15 (4): 1137-1187.

Ang, A., y Bekaert, G. (2002b). Regime Switches in Interest Rates. Journal of Business \& Economic Statistics, 20 (2): 163-182.

Ang, A., y Bekaert, G. (2002c). Short rate nonlinearities and regime switches. Journal of Economic Dynamics and Control, 26 (7-8): 1243-1274.

Ang, A., y Bekaert, G. (2004). How regimes affect asset allocation. Financial Analysts Journal, 60 (2): 86-99.

Areal, N., Cortez, M. C., y Silva, F. (2013). The conditional performance of US mutual funds over different market regimes: do different types of ethical screens matter? Financial Markets and Portfolio Management, 27 (4): 397-429.

Bank for International Settlements. (2016). Triennial Central Bank Survey of foreign exchange and OTC derivatives markets in 2016. Recuperado at 12 de diciembre de 2018, a partir de: http://www.bis.org/publ/rpfx16. $\mathrm{htm} ? \mathrm{~m}=6 \% 7 \mathrm{C} 35$

Bauwen, L., Hafner, C. M., y Rombouts, J. V. K. (2007). Multivariate mixed normal conditional heteroskedasticity. Computational Statistics \& Data analysis, 51 (7): 3551-3566.

Bezerra, P. C. S., y Albuquerque, P. H. M. (2017). Volatility forecasting via SVR-GARCH with mixture of Gaussian kernels. Computational Management Science, 14 (2): 179-196.

Black, F., y Litterman, R. (1992). Global portfolio optimization. Financial Analysts Journal, 48 (5): 28-43.

Bodie, Z., Kane, A., y Marcus, A. (2014). Investments global edition (10th ed.). New York, USA: Mc Graw-Hill.

Bollerslev, T. (1987). A Conditionally Heteroskedastic time series model for speculative prices and rates of return. The Review of Economics and Statistics, 69 (3): 542-547.

Brooks, C., y Persand, G. (2001). The trading profitability of forecasts of the gilt-equity yield ratio. International journal of forecasting, 17 (1): 11-29.

Buckley, I., Saunders, D., y Seco, L. (2008). Portfolio optimization when asset returns have the Gaussian mixture distribution. European Journal of Operational Research, 185 (3): 1434-1461.

Cabrera, G., Coronado, S., Rojas, O., y Venegas-Martínez, F. (2017). Synchronization and Changes in Volatilities in the Latin American'S Stock Exchange Markets. International Journal of Pure and Applied Mathematics, 114 (1).

Camacho, M., y Perez-Quiros, G. (2014). Commodity Prices and the Business Cycle in Latin America: Living and Dying by Commodities? Emerging Markets Finance and Trade, 50 (2): 110-137.

Castellano, R., y Scaccia, L. (2014). Can CDS indexes signal future turmoils in the stock market? A Markov switching perspective. CEJOR, 22 (2): 285-305.

Chung, S. K. (2009). Bivariate mixed normal GARCH models and out-of-sample hedge performances. Finance Research Letters, 6 (3): 130-137.

Dark, J. (2015). Futures hedging with Markov switching vector error correction FIEGARCH and FIAPARCH. Journal of Banking and Finance, 61 : S269-S285.

De la Torre, O., Galeana-figueroa, E., y Álvarez-García, J. (2018). Using Markov-Switching models in Italian , British , U . S . and Mexican equity portfolios : a performance test. Electronic Journal of Applied Statistical Analysis, 11 (2): 489-505.

Dempster, A. P., Laird, N. M., y Rubin, D. B. (1977). Maximum Likelihood from Incomplete Data via the EM Algorithm. Journal of the Royal Statistical Society. Series B (Methodological), 39 : 1-38.

Dubinskas, P., y Stungurienè, S. (2010). Alterations in the financial markets of the baltic countries and Russia in the period of Economic cownturn. Technological and Economic Development of Economy, 16 (3): 502-515.

Dueker, M. (1997). Markov Switching in GARCH Processes and Mean- Reverting Stock-Market Volatility. Journal of business \& Economics Statistics, 15 (1): 26-34. 
Dufrénot, G., Mignon, V., y Péguin-Feissolle, A. (2011). The effects of the subprime crisis on the Latin American financial markets: An empirical assessment. Economic Modelling, 28 (5): 2342-2357.

Engle, R. (1982). Autoregressive Conditional Heteroscedasticity with estimates of the variance of United Kingdom inflation. Econometrica, 50 (4): 987-1007.

Fama, E. (1965). The behavior of stock-market prices. Journal of business, 38 (1): 34-105.

Fama, E. F. (1963). Mandelbrot and the Stable Paretian Hypothesis. The journal of business, 36 (4): 420-429.

Geweke, J., y Amisano, G. (2011). Hierarchical Markov normal mixture models with applications to financial asset returns. Journal of Applied Econometrics, 26 (1): 1-29.

Girdzijauskas, S., Štreimikienė, D., Čepinskis, J., Moskaliova, V., Jurkonytė, E., y Mackevičius, R. (2009). Formation of Economic bubles: cuases and possible interventions. Technological and Economic Development of Economy, 15 (2): 267-280.

Glosten, L., Jaganathan, R., y Runkle, D. E. (1993). On the Relation between the Expected Value and the Volatility of the Nominal Excess Return on Stocks. The Journal of Finance, 48 (5): 1779-1801.

Haas, M, Mittnik, S., y Paolella, M. S. (2004). Mixed normal conditional heteroskedasticity. Journal of financial Econometrics, 2 (2): 211-250.

Haas, Markus, Mittnik, S., y Paolella, M. S. (2004). A New Approach to Markov-Switching GARCH Models. Journal of financial Econometrics, 2 (4): 493-530.

Hamilton, J. D. (1989). A New Approach to the Economic Analysis of Nonstationary Time Series and the Business Cycle. Econometrica, 57 (2): 357-384.

Hamilton, J. D. (1990). Analysis of time series subject to changes in regime. Journal of Econometrics, 45 (1-2): $39-70$.

Hamilton, J. D. (1994). Time Series Analysis. Princeton: Princeton university press.

Hamilton, J. D., y Susmel, R. (1994). Autoregressive conditional heteroskedasticity and changes in regime. Journal of Econometrics, 64 (1-2): 307-333.

Hauptmann, J., Hoppenkamps, A., Min, A., Ramsauer, F., y Zagst, R. (2014). Forecasting market turbulence using regime-switching models. Financial Markets and Portfolio Management, 28 (2): 139-164.

Ishijima, H., y Uchida, M. (2011). The Regime Switching Portfolios. Asia-Pacific Financial Markets, 18 (2): $167-$ 189.

Jorion, P. (1992). Portfolio optimization in practice. Financial analysts journal, 48 (1): 68-74.

Kanas, A. (2005). Regime linkages between the Mexican currency market and emerging equity markets. Economic Modelling, 22 (1): 109-125.

Kim, C.-J. (1994). Dynamic linear models with Markov-switching. Journal of Econometrics, 60 (1-2): 1-22.

Klaassen, F. (2002). Improving GARCH volatility forecasts with regime-switching GARCH. En Advances in Markov-Switching Models (223-254). Heidelberg: Physica-Verlag HD.

Klein, A. C. (2013). Time-variations in herding behavior: Evidence from a Markov switching SUR model. Journal of International Financial Markets, Institutions \& Money, 26 : 291-304.

Kritzman, M., Page, S., y Turkington, D. (2012). Regime Shifts: Implications for Dynamic Strategies. Financial Analysts Journal, 68 (3): 22-39.

Kutty, G. (2010). the Relationship Between Exchange Rates and Stock Prices : the Case of Mexico. North American Journal of Finance and Banking Research, 4 (4): 1-12.

Lamoureux, C. G., y Lastrapes, W. D. (1990). Persistence in Variance, Structural Change, and the GARCH Model. Journal of Business \& Economic Statistics, 8 (2): 225-234.

Levy, M., y Kaplanski, G. (2015). Portfolio selection in a two-regimeworld. European Journal of Operational Research, 242 (2): 514-524.

Lopes, J. M., y Nunes, L. C. (2012). A Markov regime switching model of crises and contagion: The case of the Iberian countries in the EMS. Journal of Macroeconomics, 34 : 1141-1153.

Ma, J., Deng, X., Ho, K.-C., y Tsai, S.-B. (2018). Regime-Switching Determinants for Spreads of Emerging Markets Sovereign Credit Default Swaps. Sustainability, 10 (2730): 1-17. 


\section{O. V. De la Torre-Torres / Contaduría y Administración 66(1), 2021, 1-32}

http://dx.doi.org/10.22201/fca.24488410e.2020.2657

Maggin, J. L., Tuttle, D., Pinto, J., y McLeavey, D. W. (2007). Managing Investment Portfolios: A Dynamic Process. (John Miley and Sons Inc, Ed.). Hoboken, USA.

Markowitz, H. (1959). Portfolio selection. Efficient diversification of investments. New Haven: Yale University Press.

Markowitz, Harry. (1952). Portfolio selection. The Journal of Finance, 7 (1): 77-91.

Markowitz, Harry. (1956). The optimization of quadratic functions subject to linear constraints. Naval research logistic quarterly, 3 (March-June): 1-113.

Michaud, R. (1989). The Markowitz Optimization Enigma: Is optimized optimal? Financial Analysts Journal, 45 (1): 31-42.

Michaud, R., y Michaud, R. (2008). Efficient asset management. A practical guide to stock portfolio optimization and asset allocation. New York: Oxford university press.

Miles, W., y Vijverberg, C.-P. (2011). Formal targets, central bank independence and inflation dynamics in the UK: A Markov-Switching approach. Journal of Macroeconomics, 33 : 644-655.

Mouratidis, K., Kenourgios, D., Samitas, A., y Vougas, D. (2013). Evaluating currency crises: A multivariate markov regime switching approach*. Manchester School, 81 (1): 33-57.

MSCI Inc. (2018). MSCI Global Investable Market Indexes Methodology. Recuperado at 2 de mayo de 2018, a partir de: http://www.msci.com/eqb/methodology/meth_docs/MSCI_Jan2015_GIMIMethodology_vf.pdf

Nelson, D. B. (1991). Conditional Heteroskedasticity in Asset Returns: A New Approach. Econometrica, 59 (2): 347.

Nikolaev, N. Y., Boshnakov, G. N., y Zimmer, R. (2013). Heavy-tailed mixture GARCH volatility modeling and Value-at-Risk estimation. Expert Systems with Applications, 40 (6): 2233-2243.

Parikakis, G. S., y Merika, A. (2009). Evaluating volatility dynamics and the forecasting ability of Markov switching models. Journal of Forecasting, 28 (8): 736-744.

Refinitiv. (2018a). Lipper Fund Research | Refinitiv. Recuperado at 27 de noviembre de 2019, a partir de: https:// www.refinitiv.com/en/products/lipper-fund-research/?utm_content=Product Name-LATAM-AMER-G-ENExact\&utm_medium=cpc\&utm_source=google\&utm_campaign=68832_RefinitivBAUPaidSearch\&elqCampaignId=5917\&utm_term=lipper mutual fund\&\&gclid=Cj0KCQiA2vjuB

Refinitiv. (2018b). Refinitiv Eikon. Recuperado at 3 de junio de 2019, a partir de: https://eikon.thomsonreuters. com/index.html

Rotta, P. N., y Valls Pereira, P. L. (2016). Analysis of contagion from the dynamic conditional correlation model with Markov Regime switching. Applied Economics, 48 (25): 2367-2382.

Sharpe, W. (1963). A simplified model for portfolio analysis. Management Science, 9 (2): 277-293.

Sharpe, W. (1964). Capital asset prices: A theory of market equilibrium under conditions of risk. The journal of finance, XIX (3): 425-442.

Sosa, M., Ortiz, E., y Cabello, A. (2018). Dynamic Linkages between Stock Market and Exchange Rate in mila Countries: A Markov Regime Switching Approach (2003-2016). Análisis Económico, 33 (83): 57-74.

Tobin, J. (1958). Liquidity preference as behavior toward risk. The Review of Economic Studies, XXV (1): 65-86.

Viterbi, A. (1967). Error bounds for convolutional codes and an asymptotically optimum decoding algorithm. IEEE Transactions on Information Theory, 13 (2): 260-269.

Walid, C., Chaker, A., Masood, O., y Fry, J. (2011). Stock market volatility and exchange rates in emerging countries: A Markov-state switching approach. Emerging Markets Review, 12 : 272-292.

Walid, C., y Duc Khuong, D. (2014). Exchange rate movements and stock market returns in a regime-switching environment: Evidence for BRICS countries. Research in International Business and Finance, (31): 46-56.

World Bank. (2019). GDP (current US\$). Recuperado at 3 de julio de 2019, a partir de: https://data.worldbank.org/ indicator/NY.GDP.MKTP.CD?locations=IN\&view=map

Xiao, Y., y Valdez, E. a. (2015). A Black-Litterman asset allocation model under Elliptical distributions. Quantitative Finance, 15 (3). 
http://dx.doi.org/10.22201/fca.24488410e.2020.2657

Zhao, H. (2010). Dynamic relationship between exchange rate and stock price: Evidence from China. Research in International Business and Finance, 24 (2): 103-112.

Zheng, T., y Zuo, H. (2013). Reexamining the time-varying volatility spillover effects: A Markov switching causality approach. North American Journal of Economics and Finance, 26 : 643-662. 